\section{Commentary: The pressure to treat pulmonary artery hypertension}

\author{
Andrew F. Feczko, MD, and Siva Raja, MD, PhD
}

Pulmonary artery hypertension (PAH) is a severe health condition affecting 20 to 70 million people worldwide. ${ }^{1,2}$ Multiple different etiologies can lead to PAH and current therapies for the disease focus on modulating signaling pathways that lead to the vasoconstriction of PAs already narrowed by medial hypertrophy and neointimal proliferation. ${ }^{2,3}$ These therapies have improved patient symptoms without decreasing mortality (approximately $10 \%$ per year) or reversing the changes associated with the disease. ${ }^{1,2}$ The battle to find a cure for this chronic condition rages on.

In the study by Miao and colleagues, ${ }^{4}$ combinations of known proangiogenic (eg, hepatocyte growth factor and vascular endothelial growth factor) and vascular stabilization factors (eg, angiopoietin 1, platelet-derived growth factors, and transforming growth factor beta) were transfected into a rat model of monocrotaline-induced PAH using a lentivirus vector. ${ }^{4}$ They hypothesized that stable neoangiogenesis would demonstrate improvement in the hemodynamic parameters associated with the disease. Through a variety of immunohistochemical and perfusion-based techniques, the authors tested different permutations of angiogenesis and stabilization factors to determine the value (if any) of their combination. The combinations of hepatocyte growth factor + angiopoietin 1 and vascular endothelial growth factor + angiopoietin 1 were shown to promote the development of increased numbers of histologically mature blood vessels. These combinations also showed improved function, with lower PA pressures and decreased right ventricular weights (a surrogate for right ventricular hypertrophy), compared with controls at 4 weeks.

\footnotetext{
From the Department of Thoracic and Cardiovascular Surgery, Heart, Vascular, and Thoracic Institute, Cleveland Clinic Foundation, Cleveland, Ohio.

Disclosures: The authors reported no conflicts of interest.

The Journal policy requires editors and reviewers to disclose conflicts of interest and to decline handling or reviewing manuscripts for which they may have a conflict of interest. The editors and reviewers of this article have no conflicts of interest.

Received for publication April 27, 2020; revisions received April 27, 2020; accepted for publication April 27, 2020; available ahead of print May 11, 2020.

Address for reprints: Siva Raja, MD, PhD, Department of Thoracic and Cardiovascu-

lar Surgery, Heart, Vascular, and Thoracic Institute, Cleveland Clinic Foundation,

J4-1, 9500 Euclid Ave, Cleveland, OH 44195 (E-mail: rajas@ccf.org).

J Thorac Cardiovasc Surg 2021;161:e435-6

0022-5223/\$36.00

Copyright (C) 2020 Published by Elsevier Inc. on behalf of The American Association for Thoracic Surgery

https://doi.org/10.1016/j.jtcvs.2020.04.131
}

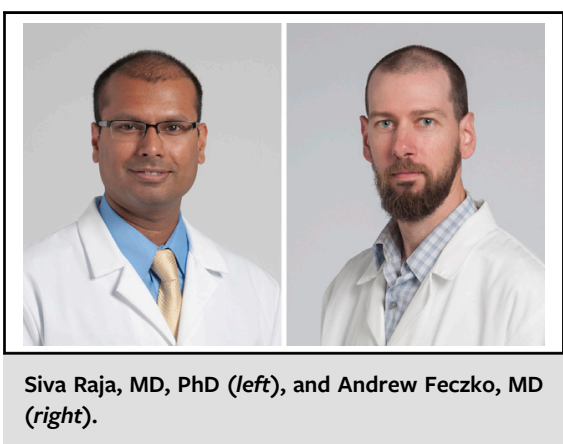

CENTRAL MESSAGE

Neoangiogenesis is a novel
treatment for pulmonary artery
hypertension but is it ready for
prime time?

By building off prior investigations by Zhang and colleagues, ${ }^{5}$ this study suggests the possibility of leapfrogging the biology underlying PAH; that is, using stable neoangiogenesis to bypass the hypertensive vasculature and reverse the cardiopulmonary effects of the disease. These results stand firmly in the realm of basic science but there are a number of issues that temper initial enthusiasm. The monocrotaline induction model of PAH, although inexpensive and easy to use, is a notably reversible and variable animal model, potentially promoting a positive result in a limited representation of the disease. ${ }^{6}$ Moreover, the rapid induction and resolution of PAH with the experimental treatment may not accurately reflect the potential for stable neoangiogenesis to reverse the chronic hemodynamic/functional changes in PAH. Lastly, this model is only 1 of many approximating PAH and may not capture all of the mechanisms underlying PAH.

Nevertheless, a journey of 1000 miles begins with a single step, and if you cannot improve the system, build a new system. Miao and colleagues ${ }^{4}$ suggest a potentially novel approach to PAH, but in doing so they have embarked on an arduous path to move from an interesting idea to a real paradigm shift. The authors will need to show long-term and durable results from this technique as well as demonstrate the specificity of their vector for the development of pulmonary vasculature, addressing the possibility of unwanted angiogenesis in other tissues. Once over these hurdles, their perseverance will be rewarded with the technical, ethical, and regulatory challenges of applying these gene-editing therapies in humans and finding out if these results hold true from rat to human. Although the work by Miao and 
colleagues $^{4}$ is preliminary, they should be recognized for contributing to the foundation on which new treatments can be built. With increasing numbers of patients being diagnosed with $\mathrm{PAH}$, the pressure to find effective treatments remains high.

\section{References}

1. Rich S, Haworth SG, Hassoun PM, Yacoub MH. Pulmonary hypertension: the unaddressed global health burden. Lancet Respir Med. 2018;2600:577-9.

2. Wilkins MR, Aman J, Harbaum L, Ulrich A, Wharton J, Rhodes CJ. Recent advances in pulmonary arterial hypertension. F1000 Res. 2018;7:1-10.
3. Ghataorhe P, Rhodes CJ, Harbaum L, Attard M, Wharton J, Wilkins MR. Pulmonary arterial hypertension-progress in understanding the disease and prioritizing strategies for drug development. J Intern Med. 2017;282:129-41.

4. Miao H, Qui F, Zhu L, Jiang B, Yuan Y, Huang B, et al. Novel angiogenesis strategy to ameliorate pulmonary hypertension. J Thorac Cardiovasc Surg. 2021;161: e417-34.

5. Zhang Y, Zhang F, Wang X, Xie Y, Du J. Sequential and timely transfection of hepatocyte growth factor and monocyte chemotactic protein-1 ameliorates hyperkinetic pulmonary artery hypertension in rabbits. J Thorac Cardiovasc Surg. 2015; 150:634-43.e2.

6. Stenmark KR, Meyrick B, Galie N, Mooi WJ, Mcmurtry IF. Animal models of pulmonary arterial hypertension: the hope for etiological discovery and pharmacological cure. Am J Physiol Lung Cell Mol Physiol. 2009;279:L1013-32.
See Article page e417.

\section{Commentary: A new treatment strategy for pulmonary arterial hypertension}

\author{
Hiroshi Date, MD
}

Despite remarkable improvements in understanding the pathobiology ${ }^{1}$ and medical treatment for pulmonary arterial hypertension (PAH), it remains to be a severe and progressive disease. Pathologically, $\mathrm{PAH}$ is associated with a severe arteriopathy leading to small vascular occlusion, increased pulmonary artery pressure, right ventricular failure, and death. Current therapies are mainly targeted to 3 pathways: prostacyclin, nitric oxide, and endothelin. These therapies are very effective and have markedly improved the prognosis of patients with PAH, including idiopathic PAH. However, these therapies are not to cure PAH but to improve pulmonary hemodynamics by dilating pulmonary small vessels. When all medical treatment fails, lung transplantation remains to be the last hope.

In this issue of the Journal, Miao and colleagues ${ }^{2}$ report a rat experimental study to develop a new treatment strategy for PAH. ${ }^{3}$ The purpose of this study was to select a suitable

From the Department of Thoracic Surgery, Graduate School of Medicine, Kyoto University, Kyoto, Japan.

Disclosures: Author has nothing to disclose with regard to commercial support.

Received for publication March 29, 2020; revisions received March 29, 2020; accepted for publication March 30, 2020; available ahead of print April 11, 2020. Address for reprints: Hiroshi Date, MD, Department of Thoracic Surgery, Graduate School of Medicine, Kyoto University, 54 Kawahara-cho, Shogoin, Sakyo-ku, Kyoto 606-8507, Japan (E-mail: hdate@kuhp.kyoto-u.ac.jp).

J Thorac Cardiovasc Surg 2021;161:e436-7

$0022-5223 / \$ 36.00$

Copyright (c) 2020 by The American Association for Thoracic Surgery

https://doi.org/10.1016/j.jtcvs.2020.03.127
Check for updates

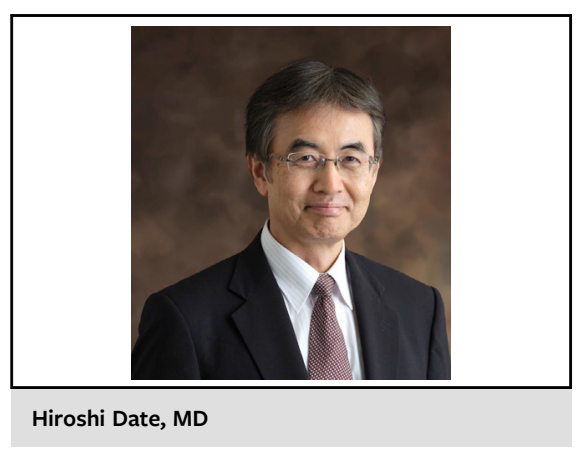

CENTRAL MESSAGE

The combination of proangiogenic and vascular stabilization

factor transfection may promote

angiogenesis and vascular matu-

ration and thus may be a new

treatment for pulmonary arterial

hypertension.

combination of angiogenic and vascular stabilization factors to improve the proliferation and maturity of neovascularization of lung tissue. The contrast medium filling time and right pulmonary artery root diameter and hemodynamic parameters in hepatocyte growth factor + angiopoietin-1 (Ang-1) and vascular endothelial growth factor + Ang-1 groups were significantly decreased compared with the vehicle group. The authors concluded that hepatocyte growth factor + Ang-1 transfection and vascular endothelial growth factor + Ang-1 transfection alleviate PAH by promoting maturation and stability of new blood vessels, 